\title{
Existence and Uniqueness of Solutions of Nonlocal Problems for Hyperbolic Equation $u_{x t}=F\left(x, t, u, u_{x}\right)$
}

\author{
L. BYSZEWSKI \\ Department of Applied Mathematics \\ Florida Institute of Technology \\ Melbourne, Florida 32901, USA
}

\begin{abstract}
The aim of the paper is to give two theorems about existence and uniqueness of continuous solutions for hyperbolic nonlinear differential problems with nonlocal conditions in bounded and unbounded domains. The results obtained in this paper can be applied in the theory of elasticity with better effect than analogous known results with classical initial conditions.
\end{abstract}

Key words: Nonlinear hyperbolic problems, nonlocal conditions, integral equations, existence and uniqueness, Banach theorem.

AMS (MOS) subject classification: $35 \mathrm{~L} 70,35 \mathrm{~L} 20,35 \mathrm{~L} 10$, $35 \mathrm{~L} 99$.

\section{INTRODUCTION.}

In this paper, we prove two theorems on existence and uniqueness of solutions of differential problems with nonlinear hyperbolic equations and with nonlocal conditions. The differential problems considered here are of the following forms:

$$
\begin{aligned}
& u_{x t}(x, t)=F\left(x, t, u(x, t), u_{x}(x, t)\right),(x, t) \in D, \\
& u(x, 0)+\sum_{i=1}^{p} h_{i}\left(x, T_{i}\right) u\left(x, T_{i}\right)=\phi(x), x \in[0, a] \\
& u(0, t)=\psi(t), t \in[0, T], \\
& \psi(0)+\sum_{i=1}^{p} h_{i}\left(0, T_{i}\right) \psi\left(T_{i}\right)=\phi(0) \\
& u_{x t}(x, t)=F\left(x, t, u(x, t), u_{x}(x, t)\right),(x, t) \in \Omega, \\
& u(x, 0)+\sum_{i=1}^{p} h_{i}\left(x, T_{i}\right) u\left(x, T_{i}\right)=\phi(x), x \in[0, a] \\
& u(0, t)=\psi(t), t \in[0, \infty)
\end{aligned}
$$

and 


$$
\psi(0)+\sum_{i=1}^{p} h_{i}\left(0, T_{i}\right) \psi\left(T_{i}\right)=\phi(0)
$$

where $\quad D:=[0, a] \times[0, T], \quad \Omega:=[0, a] \times[0, \infty), \quad T_{i} \quad(i=1, \quad \ldots, p)$ are finite numbers such that $0<T_{1}<T_{2}<\ldots<T_{p} \leq T$ and $F, \phi, \psi, h_{i}(i=1, \ldots, p)$ are given functions satisfying some assumptions.

For arbitrary domain $\Delta \subset \mathbb{R}^{2}$ denote by $C^{1,2}[\Delta, \mathbb{R}]$ the set of all continuous functions $w: \Delta \rightarrow \mathbb{R}$ such that the derivatives $w_{t}, w_{x}$ and $w_{t x}$ are continuous in $\Delta$.

We seek unique solutions of problems $(1)-(4)$ and $\left(1^{1}\right)-\left(4^{1}\right)$ in the class $C^{1,2}[D, \mathbb{R}]$ and $C^{1,2}[\Omega, \mathbb{R}]$, respectively.

The results obtained in this paper are direct generalizations of Theorems 1 and 3 given by Chi, Poorkarimi, Wiener and Shah in [3] and include the results of those theorems. If the functions $h_{i}$ $(i=1, \ldots, p)$ from condition (2) considered in the paper are identically equal zero then problems (1)-(4) and $\left(1^{1}\right)-\left(4^{1}\right)$ from this paper are reduced to problems (1), (2) and (1), (7), respectively, from [3].

Theorems 1 and 2 from the paper can be applied in the theory of elasticity with better effect than analogous known theorems with classical initial conditions. Namely, the following sum:

$$
u(x, 0)+\sum_{i=1}^{p} h_{i}\left(x, T_{i}\right) u\left(x, T_{i}\right) \text { for } x \in[0, a]
$$

is more precise to measurement of a state of a vibrating system then the only one measurement

$$
u(x, 0) \text { for } x \in[0, a]
$$

of the state of the vibrating system.

The results of this paper are also some generalizations of those given by Ladde, Lakshmikantham, and Vatsala [5] (see [5], Sections 5.3 and 5.4), and by Krzyzanski [4] (see [4], Section 77.2).

Nonlinear differential problems of parabolic type with nonlocal inequalities of type (2) and a physical interpretation of these inequalities were considered by the author in [1]. Condition (2) was introduced the first time for linear parabolic problems by Chabrowski in [2].

\section{THEOREMS.}

Theorem 1. Assume that

(i) $\quad \phi \in C^{1}([0, a], \mathbb{R}), \psi \in C^{1}([0, T], \mathbb{R}), h_{i} \in C^{1}(D, \mathbb{R})(i=1, \ldots, p)$.

(ii) There is a constant $0<K<\frac{1}{3 p}$ such that

$$
\left|h_{i}(x, t)\right| \leq K \text { and }\left|\left[h_{i}(x, t)\right]_{x}\right| \leq K \text { for }(x, t) \in D(i=1, \ldots, p) .
$$

(iii) $F \in C\left(D \times \mathbb{R}^{2}, \mathbb{R}\right)$ and there is a positive constant $L$ such that $F$ satisfies the following Lipschitz condition 


$$
|F(x, t, z, q)-F(x, t, \bar{z}, \bar{q})| \leq L(|z-\bar{z}|+|q-\bar{q}|)
$$

for $z, \bar{z}, q, \bar{q} \in \mathbb{R}$ uniformly with respect to $(x, t) \in D$.

(iv) $C$ is a positive constant such that $C>\frac{L(a+1)}{1-3 K p}$.

Then problem (1)-(4) has a unique solution belonging to $C^{1,2}[D, \mathbb{R}]$.

Proof. It is evident that if the function $u \in C^{1,2}[D, \mathbb{R}]$ satisfies problem (1) - (4), then it also satisfies the integral differential equation

$$
\begin{aligned}
u(x, t)=\phi(x)-\phi(0) & +\psi(t)-\sum_{i=1}^{p} h_{i}\left(x, T_{i}\right) u\left(x, T_{i}\right)+\sum_{i=1}^{p} h_{i}\left(0, T_{i}\right) u\left(0, T_{i}\right) \\
& +\int_{0}^{x} \int_{0}^{t} F\left(\xi, \eta, u(\xi, \eta), u_{\xi}(\xi, \eta)\right) d \eta d \xi .
\end{aligned}
$$

Conversely, if the function $u \in C^{1}(D, \mathbb{R})$ and satisfies equation (7), then it has continuous derivative $u_{x t}=u_{t x}$ in $D$, satisfies equation (1) and, moreover, conditions (2) - (4). Therefore, we will seek the solution of equation (7). For this purpose we introduce the operator

$$
\begin{aligned}
(A w)(x, t):=\phi(x)- & \phi(0)+\psi(t)-\sum_{i=1}^{p} h_{i}\left(x, T_{i}\right) w\left(x, T_{i}\right)+\sum_{i=1}^{p} h_{i}\left(0, T_{i}\right) w\left(0, T_{i}\right) \\
& +\int_{0}^{x} \int_{0}^{t} F\left(\xi, \eta, w(\xi, \eta), w_{\xi}(\xi, \eta)\right) d \eta d \xi
\end{aligned}
$$

on the space $C^{1}(D, \mathbb{R})$.

We use a weighted norm in $C^{\mathbf{l}}(D, \mathbb{R})$ (compare [3]) given by the formula

$$
\|w\|=\max _{(x, t) \in D} e^{-C t}\left(|w(x, t)|+\left|w_{x}(x, t)\right|\right) .
$$

Since $\phi \in C^{\mathbf{1}}([0, a], \mathbb{R}), \psi \in C^{\mathrm{d}}([0, T], \mathbb{R}), h_{i} \in C^{\mathrm{d}}(D, \mathbb{R})(i=1, \ldots, p)$ and $F \in C\left(D \times \mathbb{R}^{2}, \mathbb{R}\right)$, operator (8) maps $C^{1}(D, \mathbb{R})$ into $C^{1}(D, \mathbb{R})$.

Now, we will show that $A$ is a contraction on $C^{1}(D, \mathbb{R})$. To this end consider the difference

$$
\begin{aligned}
& (A w)(x, t)-(A \bar{w})(x, t) \\
& =\int_{0}^{x} \int_{0}^{t}\left[F\left(\xi, \eta, w(\xi, \eta), w_{\xi}(\xi, \eta)\right)-F\left(\xi, \eta, \bar{w}(\xi, \eta), \bar{w}_{\xi}(\xi, \eta)\right)\right] d \eta d \xi \\
& -\sum_{i=1}^{p} h_{i}\left(x, T_{i}\right)\left[w\left(x, T_{i}\right)-\bar{w}\left(x, T_{i}\right)\right]+\sum_{i=1}^{p} h_{i}\left(0, T_{i}\right)\left[w\left(0, T_{i}\right)-\bar{w}\left(0, T_{i}\right)\right]
\end{aligned}
$$

for $w, \bar{w} \in C^{1}(D, \mathbb{R})$ and apply the Lipschitz condition (6). Then

$$
\begin{gathered}
|(A w)(x, t)-(A \bar{w})(x, t)| \leq L \int_{0}^{x} \int_{0}^{t}\left(|w(\xi, \eta)-\overline{\mathrm{w}}(\xi, \eta)|+\left|w_{\xi}(\xi, \eta)-\bar{w}_{\xi}(\xi, \eta)\right|\right) d \eta d \xi \\
\quad+\sum_{i=1}^{p}\left|h_{i}\left(x, T_{i}\right)\left\|w\left(x, T_{i}\right)-\bar{w}\left(x, T_{i}\right)\left|+\sum_{i=1}^{p}\right| h_{i}\left(0, T_{i}\right)\right\| w\left(0, T_{i}\right)-\bar{w}\left(0, T_{i}\right)\right|
\end{gathered}
$$

for $w, \bar{w} \in C^{1}(D, \mathbb{R})$.

Next, consider the following difference 


$$
\begin{aligned}
& {[(A w)(x, t)]_{x}-[(A \bar{w})(x, t)]_{x}} \\
& =\int_{0}^{t}\left[F\left(x, \eta, w(x, \eta), w_{x}(x, \eta)\right)-F\left(x, \eta, \bar{w}(x, \eta), \bar{w}_{x}(x, \eta)\right)\right] d \eta \\
& -\sum_{i=1}^{p}\left[h_{i}\left(x, T_{i}\right)\right]_{x}\left[w\left(x, T_{i}\right)-\bar{w}\left(x, T_{i}\right)\right]-\sum_{i=1}^{p} h_{i}\left(x, T_{i}\right)\left[w\left(x, T_{i}\right)-\bar{w}\left(x, T_{i}\right)\right]_{x}
\end{aligned}
$$

for $w, \bar{w} \in C^{1}(D, \mathbb{R})$ and apply the Lipschitz condition (6). Then

$$
\begin{aligned}
& \left|[(A w)(x, t)]_{x}-[(A \bar{w})(x, t)]_{x}\right| \\
& \leq L \int_{0}^{t}\left(|w(x, \eta)-\bar{w}(x, \eta)|+\left|w_{x}(x, \eta)-\bar{w}_{x}(x, \eta)\right|\right) d \eta \\
& +\sum_{i=1}^{p}\left|\left[h_{i}\left(x, T_{i}\right)\right]_{x}\right|\left|w\left(x, T_{i}\right)-\bar{w}\left(x, T_{i}\right)\right|+\sum_{i=1}^{p}\left|h_{i}\left(x, T_{i}\right)\right|\left|w_{x}\left(x, T_{i}\right)-\bar{w}_{x}\left(x, T_{i}\right)\right|
\end{aligned}
$$

for $w, \bar{w} \in C^{\mathrm{l}}(D, \mathbb{R})$.

Finally, by (5) and (9), we have

$$
\begin{aligned}
& \quad \mathrm{e}^{-C t}\left[\sum _ { i = 1 } ^ { p } \left(\left|h_{i}\left(x, T_{i}\right)\right|\left|w\left(x, T_{i}\right)-\bar{w}\left(x, T_{i}\right)\right|+\left|h_{i}\left(0, T_{i}\right)\right|\left|w\left(0, T_{i}\right)-\bar{w}\left(0, T_{i}\right)\right|\right.\right. \\
& \left.\left.\quad+\left|\left[h_{i}\left(x, T_{i}\right)\right]{ }_{x}\right|\left|w\left(x, T_{i}\right)-\bar{w}\left(x, T_{i}\right)\right|+\left|h_{i}\left(x, T_{i}\right)\right|\left|w_{x}\left(x, T_{i}\right)-\bar{w}_{x}\left(x, T_{i}\right)\right|\right)\right] \\
& \leq 3 K p\|w-\bar{w}\| \text { for } w, \bar{w} \in C^{1}(D, \mathbb{R}) .
\end{aligned}
$$

Simultaneously, from [3],

$$
\begin{aligned}
& L e^{-C t} \int_{0}^{x} \int_{0}^{t}\left(|w(\xi, \eta)-\bar{w}(\xi, \eta)|+\left|w_{\xi}(\xi, \eta)-\bar{w}_{\xi}(\xi, \eta)\right|\right) d \eta d \xi \\
& +L e^{-C t} \int_{0}^{t}\left(|w(x, \eta)-\bar{w}(x, \eta)|+\left|w_{x}(x, \eta)-\bar{w}_{x}(x, \eta)\right|\right) d \eta \\
& \leq \frac{L(a+1)}{C}\|w-\bar{w}\| \text { for } w, \bar{w} \in C^{\mathbf{l}}(D, \mathbb{R}) .
\end{aligned}
$$

Therefore, by (10) - (13) and (9),

$$
\|A w-A \bar{w}\| \leq\left(\frac{L(a+1)}{C}+3 K p\right)\|w-\bar{w}\| \text { for } w, \bar{w} \in C^{1}(D, \mathbb{R}) .
$$

If we define $q=\frac{L(a+1)}{C}+3 K p$ then, by assumption (iv),

$$
\|A w-A \bar{w}\| \leq q\|w-\bar{w}\| \text { for } w, \bar{w} \in C^{\mathbf{l}}(D, \mathbb{R})
$$

with $0<q<1$. This shows that operator $A$ is a contraction. Consequently, the proof of Theorem 1 is complete.

Theorem 2. Assume that

(i) $\phi \in C^{1}([0, a], \mathbb{R}), \psi \in C^{1}([0, \infty), \mathbb{R}), h_{i} \in C^{\mathbf{l}}(\Omega, \mathbb{R})(i=1, \ldots, p)$.

(ii) There is a constant $0<K<\frac{1}{3 p}$ such that 


$$
\left|h_{i}(x, t)\right| \leq K \text { and }\left|\left[h_{i}(x, t)\right]_{x}\right| \leq K \text { for }(x, t) \in \Omega \quad(i=1, \ldots, p)
$$

(iii) $F \in C\left(\Omega \times \mathbb{R}^{2}, \mathbb{R}\right)$ and there is a positive constant $L$ such that $F$ satisfies the following Lipschitz condition

$$
|F(x, t, z, q)-F(x, t, \bar{z}, \bar{q})| \leq L(|z-\bar{z}|+|q-\bar{q}|)
$$

for $z, \bar{z}, q, \bar{q} \in \mathbb{R}$ uniformly with respect to $(x, t) \in \Omega$.

(iv) $C$ is a postive constant such that $C>\frac{L(a+1)}{1-3 K p}$.

(v) There are positive constants $K_{i}(i=1,2,3)$ such that

$$
\begin{gathered}
|\phi(x)| \leq K_{1} e^{C t}, \quad\left|\phi^{\prime}(x)\right| \leq K_{1} e^{C t} \text { for } x \in[0, a], \\
|\psi(t)| \leq K_{2} e^{C t} \text { for } t \in[0, \infty) \quad \text { and } \\
|F(x, t, 0,0)| \leq K_{3} e^{C t} \text { for }(x, t) \in \Omega .
\end{gathered}
$$

Then problem $\left(1^{1}\right)-\left(4^{1}\right)$ has a unique solution $u \in C^{1,2}[\Omega, \mathbb{R}]$ satisfying the following condition

$$
\sup _{(x, t) \in \Omega} e^{-C t}\left(|u(x, t)|+\left|u_{x}(x, t)\right|\right)<\infty .
$$

Proof. Analogously as in the proof of Theorem 1, we reduce problem $\left(1^{1}\right)-\left(4^{1}\right)$ to the integral differential equation (7) and introduce operator $(8)$ on the space $C^{1}(\Omega, \mathbb{R})$ with the norm

$$
\|w\|=\sup _{(x, t) \in \Omega} e^{-C t}\left(|w(x, t)|+\left|w_{x}(x, t)\right|\right)
$$

First, we will show that operator (8) maps $C^{1}(\Omega, \mathbb{R})$ into $C^{1}(\Omega, \mathbb{R})$. Here observe that

$$
\begin{gathered}
(A w)(x, t)=\phi(x)-\phi(0)+\psi(t)-\sum_{i=1}^{p} h_{i}\left(x, T_{i}\right) w\left(x, T_{i}\right)+\sum_{i=1}^{p} h_{i}\left(0, T_{i}\right) w\left(0, T_{i}\right) \\
+\int_{0}^{x} \int_{0}^{t}\left[F\left(\xi, \eta, w(\xi, \eta), w_{\xi}(\xi, \eta)\right)-F(\xi, \eta, 0,0)\right] d \eta d \xi \\
+\int_{0}^{x} \int_{0}^{t} F(\xi, \eta, 0,0) d \eta d \xi \text { for } w \in C^{1}(\Omega, \mathbb{R})
\end{gathered}
$$

and

$$
\begin{gathered}
{[(A w)(x, t)]_{x}=\phi^{\prime}(x)-\sum_{i=1}^{p}\left[h_{i}\left(x, T_{i}\right)\right]_{x} w\left(x, T_{i}\right)-\sum_{i=1}^{p} h_{i}\left(x, T_{i}\right)\left[w\left(x, T_{i}\right)\right]_{x}} \\
\left.+\int_{0}^{t} F\left(x, \eta, w(x, \eta), w_{x}(x, \eta)\right)-F(x, \eta, 0,0)\right] d \eta \\
\quad+\int_{0}^{t} F(x, \eta, 0,0) d \eta \text { for } w \in C^{\mathbf{l}}(\Omega, \mathbb{R}) .
\end{gathered}
$$

Hence, from (17), (18) and (15),

$$
|(A w)(x, t)|+\left|[(A w)(x, t)]{ }_{x}\right| \leq H_{1}(x, t)+H_{2}(x, t) \text { for } w \in C^{1}(\Omega, \mathbb{R}),
$$

where 


$$
\begin{gathered}
H_{1}(x, t)=|\phi(x)|+|\phi(0)|+\left|\phi^{\prime}(x)\right|+|\psi(t)| \\
+L \int_{0}^{x} \int_{0}^{t}\left(|w(\xi, \eta)|+\left|w_{\xi}(\xi, \eta)\right|\right) d \eta d \xi+\int_{0}^{x} \int_{0}^{t}|F(\xi, \eta, 0,0)| d \eta d \xi \\
+L \int_{0}^{t}\left(|w(x, \eta)|+\left|w_{x}(x, \eta)\right|\right) d \eta+\int_{0}^{t}|F(x, \eta, 0,0)| d \eta
\end{gathered}
$$

$$
\begin{aligned}
& H_{2}(x, t)=\sum_{i=1}^{p}\left|h_{i}\left(x, T_{i}\right)\right|\left|w\left(x, T_{i}\right)\right|+\sum_{i=1}^{p}\left|h_{i}\left(0, T_{i}\right)\right|\left|w\left(0, T_{i}\right)\right| \\
& +\sum_{i=1}^{p}\left|\left[h_{i}\left(x, T_{i}\right)\right]{ }_{x}\right|\left|w\left(x, T_{i}\right)\right|+\sum_{i=1}^{p}\left|h_{i}\left(x, T_{i}\right)\right|\left|\left[w\left(x, T_{i}\right)\right]{ }_{x}\right| .
\end{aligned}
$$

By, (21), (14) and (16),

$$
e^{-C t} H_{2}(x, t) \leq 3 K p\|w\| \text { for } w \in C^{\mathrm{l}}(\Omega, \mathbb{R}) \text {. }
$$

Simultaneously, from [3],

$$
e^{-C t} H_{1}(x, t) \leq K_{1}+K_{2}+\frac{K_{3}(a+1)}{C}+\frac{L(a+1)}{C}\|w\| \text { for } w \in C^{\mathrm{l}}(\Omega, \mathbb{R}) .
$$

Consequently, by (19) - (21), (23), (22) and (16),

$$
\|A w\| \leq K_{1}+K_{2}+\frac{K_{3}(a+1)}{C}+\left(\frac{L(a+1)}{C}+3 K p\right)\|w\| \text { for } w \in C^{1}(\Omega, \mathbb{R}) \text {. }
$$

This proves that operator (8) maps $C^{\mathrm{l}}(\Omega, \mathbb{R})$ into $C^{\mathrm{d}}(\Omega, \mathbb{R})$. For the proof of contraction, we repeat the corresponding computations from Theorem 1. The proof of Theorem 2 is therefore complete.

\section{ACKNOWLEDGEMENT.}

I would like to thank Professor Lakshmikantham and the Kosciuszko Foundation of New York for giving me the possibility of writing this paper during my stay at the Department of Applied Mathematics of the Florida Institute of Technology.

\section{REFERENCES.}

[1] L. Byszewski, Strong maximum and minimum principles for parabolic problems with nonlocal inequalities, Zeitschrift für Angewandte Mathematik und Mechanik 70.3 (1990), 202-205.

[2] J. Chabrowski, On non-local problems for parabolic equations, Nagoya Math. J. 93 (1984), 109-131.

[3] H. Chi, H. Poorkarimi, J. Wiener, S. M. Shah, On the exponential growth of solutions to nonlinear hyperbolic equations, International Journal of Mathematics and Mathematical Sciences 12 (1989), 539-546.

[4] M. Krzyzanski, Partial differential equations of second order, Vol. II, Polish Scientific Publishers, Warsaw, 1971.

[5] G.S. Ladde, V. Lakshmikantham, A.S. Vatsala, Monotone iterative techniques for nonlinear differential equations, Pitman Advanced Publishing Program, Boston, London, Melbourne, 1985. 


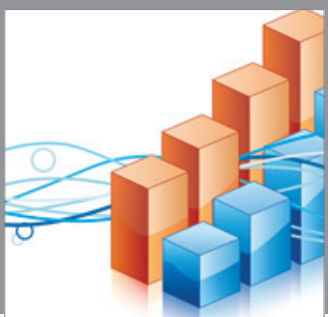

Advances in

Operations Research



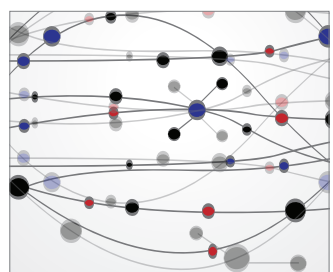

\section{The Scientific} World Journal
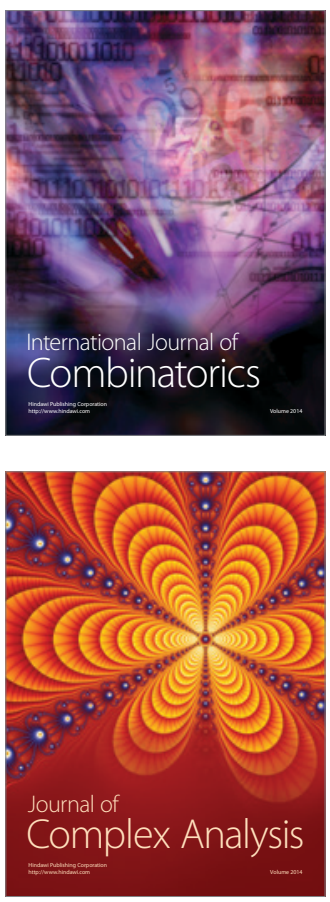

International Journal of

Mathematics and

Mathematical

Sciences
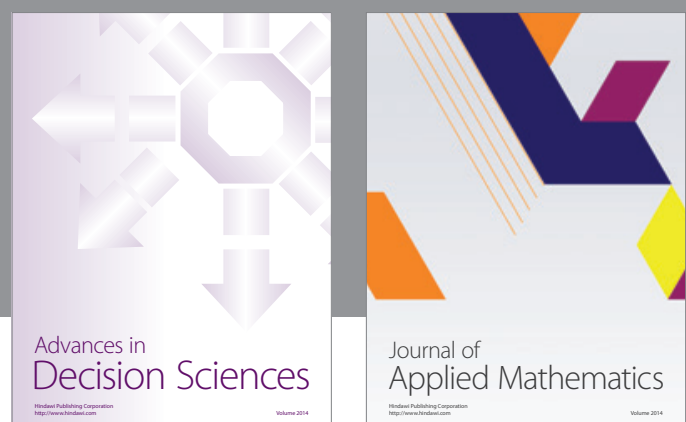

Journal of

Applied Mathematics
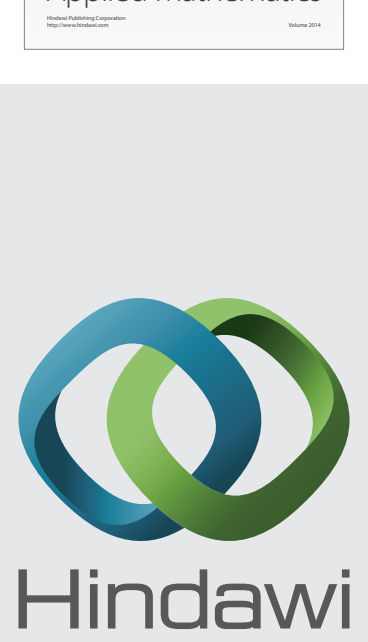

Submit your manuscripts at http://www.hindawi.com
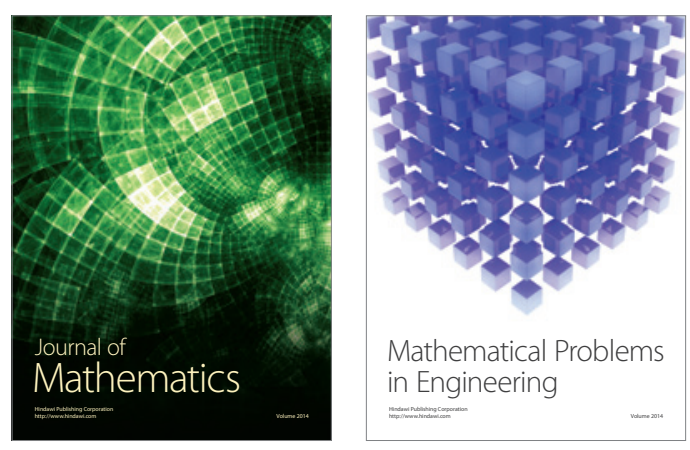

Mathematical Problems in Engineering
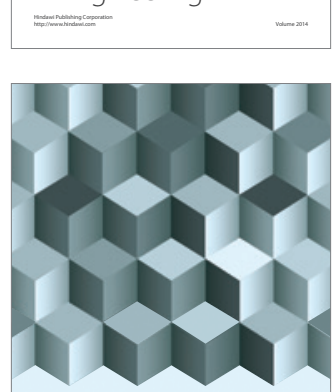

Journal of

Function Spaces
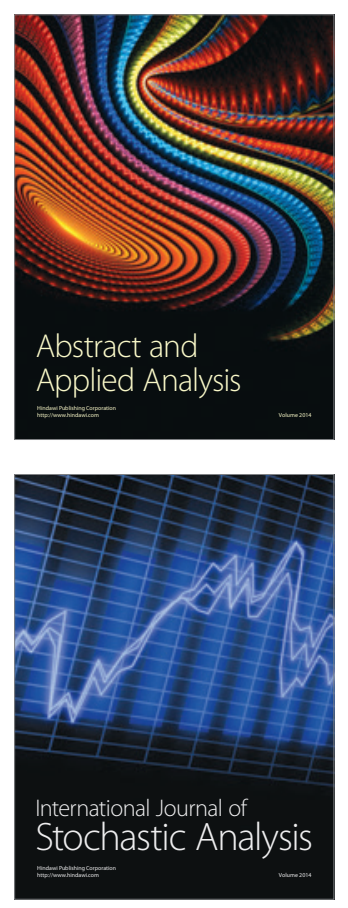

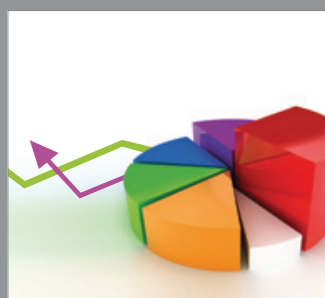

ournal of

Probability and Statistics

Promensencen
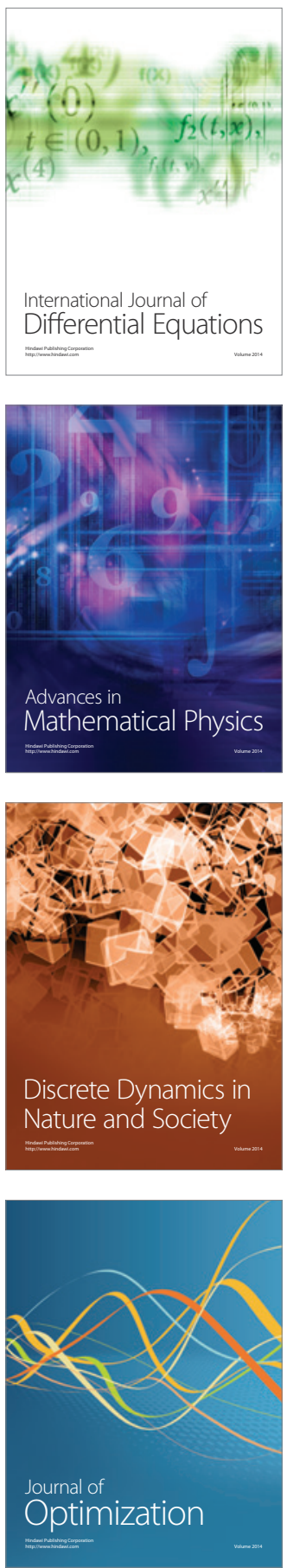